\title{
Splanchnic haemodynamic disturbances in perinatal sepsis
}

\author{
S T Kempley, E Murdoch
}

Whitechapel, St

Bartholomew's and the

Royal London School

of Medicine and

Dentistry, London, UK

S T Kempley

E Murdoch

Correspondence to:

Dr Kempley, Elizabeth Ward,

Royal London Hospital,

Whitechapel, London

E1 1BB, UK

email:

Accepted 27 March 2000
Neonatal Unit, Royal London Hospital,

\begin{abstract}
Aim-To determine the effect of perinatal bacterial infection on the neonatal splanchnic circulation.

Subjects/Setting-76 premature infants with appropriate birth weight for gestation admitted for neonatal intensive care. Methods-Doppler ultrasound was used to measure blood flow velocity and pulsatility index in the superior mesenteric artery and coeliac axis during the first 24 hours of life. Babies were classified according to the results of blood and surface cultures, as well as the presence or absence of maternal prolonged membrane rupture.

Results-Infection status had a significant effect on pulsatility index in both arteries, with that in the coeliac axis being reduced from 1.27 to 0.80 in babies with infection $(p<0.0001)$. Coeliac axis blood flow velocity was significantly increased in those with infection (from 34.6 to $46.5 \mathrm{~cm} / \mathrm{s}$; p < 0.05).

Conclusion-As early as the first day of postnatal life, infected neonates show a pattern of splanchnic hyperaemia similar to that found in adult systemic inflammatory response syndrome.
\end{abstract}

(Arch Dis Child Fetal Neonatal Ed 2000;83:F139-F142)

Keywords: sepsis; splanchnic circulation; bacterial infection; infection; systemic inflammatory response syndrome

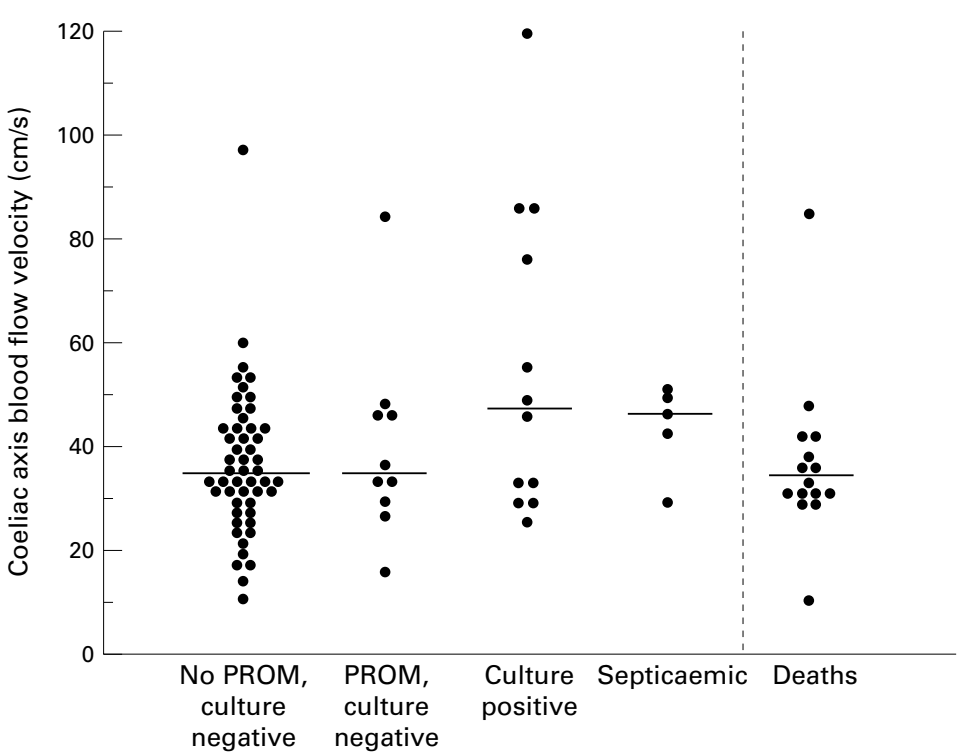

Figure 1 Coeliac axis blood flow velocity according to infection status, and in infants who died. PROM, premature rupture of membranes.
In adults, the definition of the systemic inflammatory response syndrome (SIRS) has facilitated research into the circulatory changes that accompany septic shock and acute systemic inflammation in intensive care patients. Research suggests that both splanchnic blood flow and oxygen consumption are increased in adults with SIRS. ${ }^{1}$

Factors that initiate SIRS include septicaemia, trauma, burns, and major surgery. Its characteristic features in adults are temperature change $\left(>38^{\circ} \mathrm{C}\right.$ or $\left.<36^{\circ} \mathrm{C}\right)$, tachycardia (heart rate $>90 / \mathrm{min}$ ), hyperventilation (respiratory rate $>20 / \mathrm{min}$ or $\mathrm{PaCO}_{2}<4.3 \mathrm{kPa}$ ), and abnormal peripheral white cell count ( $>12 \times 10^{9} / 1$ or $<4 \times 10^{9} / 1$ or $>10 \%$ immature forms). The process may continue even after the initiating factor is no longer present, possibly as a result of endotoxin release through a damaged gastrointestinal tract.

The definition of SIRS is not readily applicable to newborn infants whose physiological and haematological indices are very different from those in adults. Nevertheless, conditions such as neonatal necrotising enterocolitis have been shown to be associated with increased superior mesenteric artery (SMA) blood flow velocity (BFV), ${ }^{23}$ and it is possible that changes similar to those found in adult SIRS could be present in other infective conditions in the neonate. The aim of this study was to determine whether neonates with perinatal bacterial infection show evidence of splanchnic hyperaemia.

\section{Methods}

The study analysed Doppler ultrasound measurements collected during projects examining risk factors for necrotising enterocolitis, in newborn babies admitted to the neonatal units of the Royal London and King's College Hospitals. BFV (time averaged mean) and pulsatility index (PI) were measured in the coeliac axis and SMA close to their origin from the abdominal aorta as described previously, ${ }^{2}$ and only values collected in the first 24 hours of life were used. All measurements were made using a Hewlett-Packard Sonos 100 ultrasound unit with $7.5 \mathrm{MHz}$ imaging and $5.0 \mathrm{MHz}$ Doppler duplex transducer. The artery was visualised in real time, and range gating was used to sample signals from the proximal segment of the artery. All Doppler measurements were derived from the peak velocity envelope and corrected for the angle of insonation. The PI was calculated as the systolic minus diastolic velocity, divided by the mean velocity.

Babies were selected for analysis if they were premature ( $<37$ weeks gestation), results of initial cultures were available, Doppler studies 


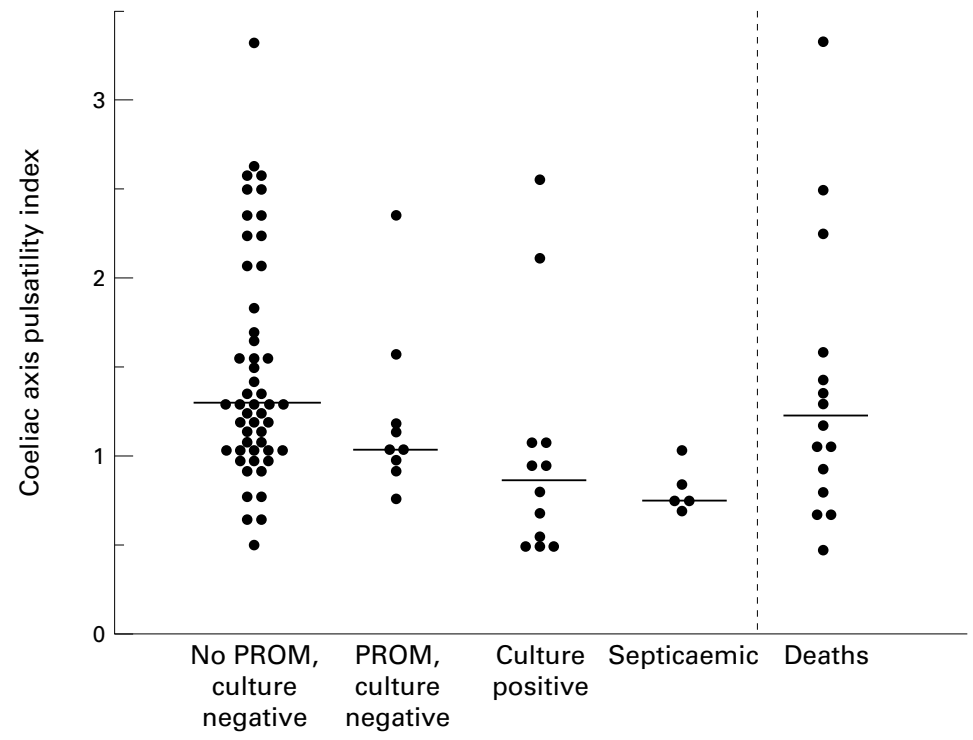

Figure 2 Coeliac axis pulsatility index according to infection status, and in infants who died. PROM, premature rupture of membranes.

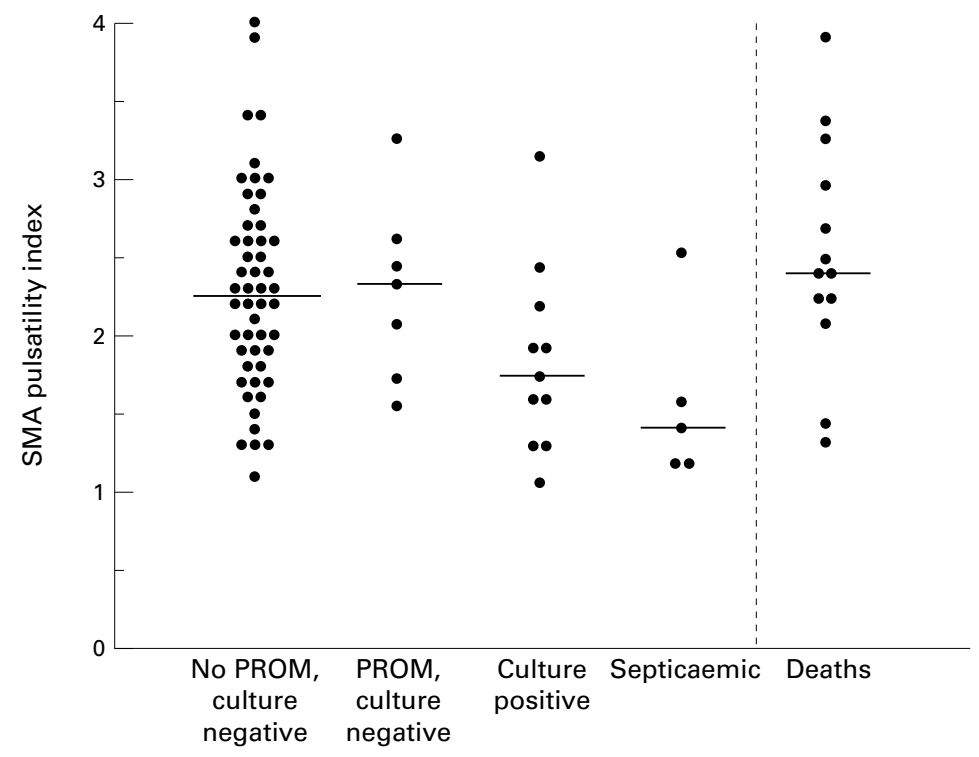

Figure 3 Superior mesenteric artery (SMA) pulsatility index according to infection status, and in infants who died. PROM, premature rupture of membranes.

were performed within the first 24 hours of life, and birth weight was above the 10th centile for gestation. Small for gestational age babies were excluded as they have previously been shown to have reduced coeliac and SMA BFV. ${ }^{4}$ (As these patients are often delivered electively by caesarean section, they have a reduced chance of having infection and their inclusion would have biased the results in favour of the hypothesis being tested.)

Patients were classified as being infected if the deep ear swab, gastric aspirate, or blood culture taken on admission to the neonatal unit yielded a positive culture. Organisms cultured included group B Streptococcus $(\mathrm{n}=5)$, various Gram negative organisms $(n=8)$, anaerobic organisms $(\mathrm{n}=2)$, Staphylococcus aureus $(\mathrm{n}=1)$, and Listeria $(\mathrm{n}=1)$. The presence of maternal prolonged membrane rupture for more than 24 hours was recorded.
Initial analysis by one way analysis of variance divided the babies into four groups:

(1) negative cultures and no prolonged rupture of membranes;

(2) prolonged rupture of membranes with negative cultures;

(3) gastric aspirate, ear swab, or umbilical swab positive with negative blood culture;

(4) positive blood cultures.

The groups were then combined and the analysis repeated, comparing babies with any positive culture (groups 3 and 4 ) with all babies with negative cultures (groups 1 and 2). In this analysis, quantitative data were assessed using the Mann-Whitney $U$ test to ensure that none of the observed differences were related to the slight skewness observed in the coeliac axis data or the presence of unbalanced groups. Qualitative data were assessed with $\chi^{2}$ or Fisher's exact test as appropriate.

To ensure that none of the effects were related to differences in birth weight, gestation, or the unit to which the babies were admitted, a multiple linear regression analysis was carried out with Doppler parameters as the dependent variable and infection, birth weight, gestation, and unit as independent variables.

\section{Results}

A significant effect of infection status on coeliac axis $\mathrm{BFV}$ (fig $1 ; \mathrm{F}=4.79, \mathrm{p}<0.01$ ), coeliac axis PI (fig $2 ; \mathrm{F}=3.19, \mathrm{p}<0.05$ ), and SMA PI (fig 3; $F=3.33, p<0.05$ ) was shown between the four groups. The effect on SMA $\mathrm{BFV}$ was not statistically significant (fig 4; $\mathrm{F}=2.37, \mathrm{p}=0.08$ ). There were no major differences in birth weight, gestation, Apgar score, ventilation, or inotrope requirement between the four groups (table 1).

Findings were very similar in uninfected babies with and without maternal prolonged membrane rupture. Infected babies with and without septicaemia had similar findings, although the highest coeliac axis BFV values were found in four infants colonised with Listeria, group B Streptococcus, Staphylococcus aureus, and a Gram negative rod. Group B Streptococcus $(\mathrm{n}=3)$ and Escherichia coli $(n=2)$ grew in cultures of blood taken from the five septicaemic infants on admission to the neonatal unit.

When the infants were simply divided into two groups according to the presence or absence of positive culture results, the infected babies had a significantly lower PI in the coeliac axis and SMA (table 2) with a significantly higher BFV in the coeliac axis. There was a non-significant trend to higher $\mathrm{BFV}$ in the SMA of infected babies.

The birth weight, gestation, and Apgar scores of these two groups were very similar, as were the physiological data at the time of measurement (table 2). There was no difference in the proportion receiving inotropes or mechanical ventilation. More of the infected babies had been born after prolonged membrane rupture. The trend towards higher mortality in the babies with negative cultures was not statistically significant. Regression analysis 


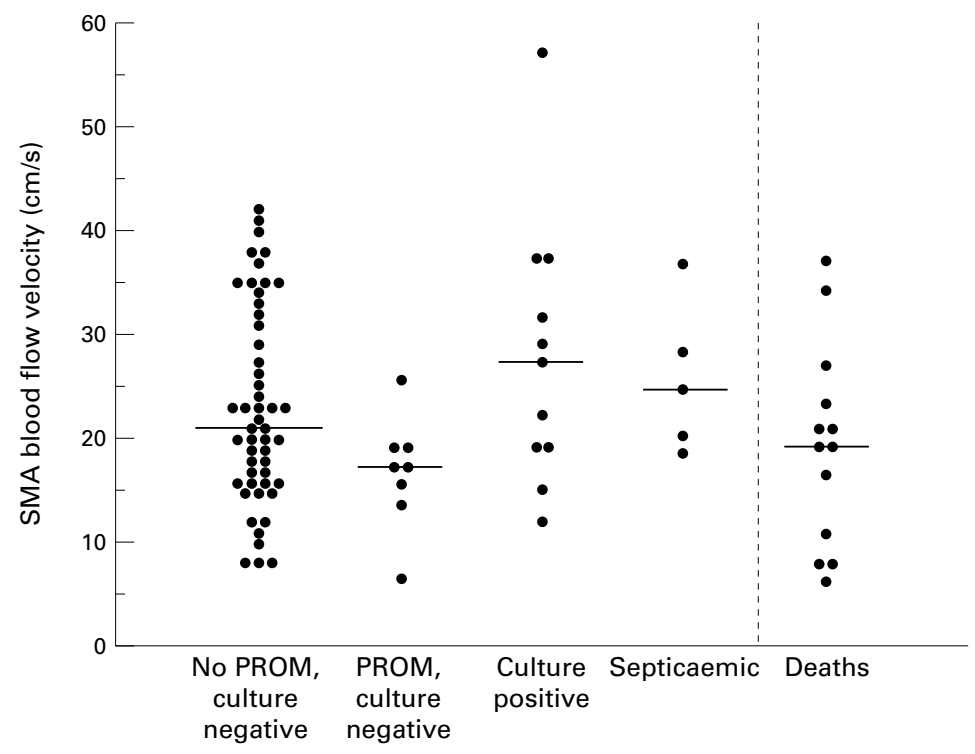

Figure 4 Superior mesenteric artery (SMA) blood flow velocity according to infection status, and in infants who died. PROM, premature rupture of membranes.

did not show any significant association of birth weight, gestation, or unit of admission with the Doppler parameters.

\section{Discussion}

As early as the first day of postnatal life, infected neonates show a pattern of splanchnic circulatory disturbance similar to that found in adult SIRS. This pattern is one in which resistance to blood flow, as indicated by pulsatility index, is reduced in the coeliac axis and SMA. This is most consistent in the coeliac axis, where there is also a definite increase in BFV.
Blood flow disturbances in babies with septicaemia were similar to those found in babies with positive surface cultures. This is not surprising, as deep ear swabs and gastric aspirate culture may disclose organisms present in amniotic fluid causing serious bacterial infection by the time of delivery. It is possible that many infants with organisms found on surface cultures also had systemic infection, as neonatal blood cultures often contain less than $0.5 \mathrm{ml}$ of blood, ${ }^{5}$ which may be insufficient to detect low colony counts of common pathogens such as group B Streptococcus or E coli. ${ }^{6}$ In adult SIRS, levels of endotoxin may be high even when blood cultures are negative. ${ }^{7}$ The very high coeliac axis BFV found in four babies colonised with Listeria, group B Streptococcus, Staphylococcus aureus, and a Gram negative organism could be the result of undetected systemic infection or the activation of systemic inflammation after the fetus has inhaled or swallowed infected amniotic fluid. The definition of SIRS in adults has allowed the study of a process that is initiated by sepsis or other insults, in which the resultant physiological disturbances may be unrelated to current blood culture results. A clinical definition of sepsis in neonates which is not solely dependent on blood culture results could facilitate research in this area.

None of the differences between the groups can be accounted for by clinical factors such as birth weight, gestation, Apgar score, or ventilatory support, nor were there any differences in physiological variables such as blood pressure or blood gases. Very few babies were being treated with inotropes. Although there was a trend towards higher mortality in babies with-

Table 1 Clinical characteristics of the four groups of patients defined according to their infection status and the presence of prolonged rupture of membranes (PROM)

\begin{tabular}{lllll}
\hline & $\begin{array}{l}\text { Culture negative, no } \\
\text { PROM }(n=51)\end{array}$ & $\begin{array}{l}\text { PROM, culture negative } \\
(n=8)\end{array}$ & $\begin{array}{l}\text { Surface culture positive } \\
(n=12)\end{array}$ & Septicaemic (n=5) \\
\hline Birth weight $(\mathrm{kg})$ & $1.14(0.51-2.40)$ & $1.47(0.54-2.47)$ & $1.01(0.67-3.10)$ & $1.29(0.57-2.52)$ \\
Gestation (weeks) & $28(23-34)$ & $29.5(24-34)$ & $27(25-36)$ & $29(24-36)$ \\
Apgar score & $8(1-10)$ & $6.5(4-10)$ & $7(2-9)$ & $9(7-9)$ \\
Inotropic support & $4(8)$ & 0 & $1(8)$ & 0 \\
Mechanical ventilation & $47(92)$ & $6(75)$ & $11(92)$ & $3(60)$ \\
\hline
\end{tabular}

Values are medians (ranges) or numbers (percentages).

Table 2 Clinical characteristics, physiological status at the time of measurement and blood flow velocity (BFV) measurements for infants with negative and positive cultures on the first day of life

\begin{tabular}{|c|c|c|c|}
\hline & Negative cultures $(n=59)$ & Infected $(n=17)$ & $p$ Value \\
\hline \multicolumn{4}{|l|}{ Clinical characteristics } \\
\hline Birth weight (kg) & $1.14(0.51-2.47)$ & $1.038(0.57-3.10)$ & \\
\hline Gestation (weeks) & $28(23-34)$ & $27(24-36)$ & \\
\hline Maternal PROM & $8(14)$ & $7(41)$ & $<0.05$ \\
\hline Five minute Apgar score & $8(1-10)$ & $8(1-10)$ & \\
\hline No with 5 min Apgar $<5$ & $5(8)$ & $1(6)$ & \\
\hline Deaths & $13(22)$ & $1(7)$ & 0.24 \\
\hline \multicolumn{4}{|c|}{ Physiological status when measurements performed } \\
\hline Age (hours) & $11(1-23)$ & $11.5(3-21)$ & \\
\hline Mean arterial $\mathrm{BP}(\mathrm{mm} \mathrm{Hg})$ & $34(10-52)$ & $35(33-44)$ & \\
\hline $\mathrm{PaCO}_{2}(\mathrm{kPa})$ & $4.7(3.8-8.5)$ & $4.2(3.2-5.4)$ & \\
\hline Inotropic support & $4(7)$ & $1(7)$ & \\
\hline Mechanical ventilation & $53(90)$ & $14(82)$ & \\
\hline \multicolumn{4}{|l|}{$B F V$ measurements } \\
\hline Coeliac axis BFV $(\mathrm{cm} / \mathrm{s})$ & $34.6(10.4-97.3)$ & $46.5(25.4-126)$ & $<0.05$ \\
\hline Coeliac axis PI & $1.27(0.54-3.32)$ & $0.80(0.48-2.55)$ & $<0.0001$ \\
\hline $\mathrm{SMA} \mathrm{BFV}(\mathrm{cm} / \mathrm{s})$ & $20.2(6.2-42.4)$ & $26.2(12.1-57.4)$ & 0.11 \\
\hline SMA PI & $2.28(1.14-3.98)$ & $1.61(1.06-3.15)$ & $<0.01$ \\
\hline
\end{tabular}

Values are given as medians (ranges) or numbers (percentages). Where no $\mathrm{p}$ value is given, the differnce is not significant. PROM, premature rupture of membranes; BP, blood pressure; PI, pulsatility index; SMA, superior mesenteric artery. 
out infection, this was not statistically significant. Perinatal bacterial infection is often expected and every baby admitted for intensive care received prophylactic systemic antibiotics to which organisms are nearly always sensitive. There was no evidence that the babies who died had a different pattern of blood flow changes from the uninfected infants (figs 1-4).

An increase in the ratio between coeliac and SMA systolic BFV has been described by Coombs et $a t^{\beta}$ in a heterogeneous group of patients at risk of necrotising enterocolitis. These patients included growth restricted infants, infants suffering from perinatal asphyxia, and infants undergoing exchange transfusion for various reasons. Their findings could mainly be explained by a reduction in SMA velocity, with no significant differences in coeliac axis BFV shown between groups. Bacteriological results were not analysed. Our findings are clearly different, as we show a significant increase in coeliac axis BFV and we specifically excluded all infants who were small for gestational age. However, it is likely that a number of insults other than infection can stimulate splanchnic hyperaemia in neonates, and some individual babies in the study of Coombs et al may have shown findings similar to ours.

BFV was significantly increased in the coeliac axis only, indicating that the inflammatory response is probably most intense and reproducible in the liver and spleen. Although the PI was reduced in the SMA, there was only a non-significant trend towards increased BFV. The SMA may be affected by a "steal" effect from the coeliac axis, the origin of which from the aorta is only a few millimetres from the SMA in the preterm neonate.

In adult SIRS, peripheral vascular resistance is decreased in the circulation as a whole ${ }^{9}$ with a paradoxical reduction in oxygen consumption. ${ }^{10}$ In contrast, splanchnic blood flow and oxygen consumption are both raised ${ }^{7}$ and are highest in those requiring noradrenaline (norepinephrine). ${ }^{1}$ Dissociation between blood flow and oxygen consumption is also demonstrated by the finding that hyperaemia is dependent on nitric oxide but increased oxygen consumption is not. ${ }^{11}$

Increased $\mathrm{BFV}$ in the coeliac axis may result in increased cardiovascular demands on severely ill neonates with infection, compromising blood flow to other organs. Despite a lowered index of vascular resistance, possibly indicating increased oxygen demand, blood flow to the bowel was not increased. In perinatal sepsis, it is therefore possible that the splanchnic circulation may contribute to cardiovascular instability, while the intestine may suffer from inadequate oxygenation if blood flow does not increase sufficiently to meet raised oxygen demands. Further studies of postnatally acquired neonatal sepsis are required to determine whether similar patterns of haemodynamic disturbance are present in this condition.

1 Meier-Hellmann A, Specht M, Hannemann L, Hassel H, Bredle DL, Reinhart K. Splanchnic blood flow is greater in septic shock treated with norepinephrine than in severe sepsis. Intensive Care Med 1996;22:1354-9.

2 Kempley ST, Gamsu HR. Superior mesenteric artery blood flow velocity in necrotising enterocolitis. Arch Dis Child 1992;67:793-6.

3 Deeg KH, Rupprecht T, Schmid E. Doppler sonographic detection of increased flow velocities in the celiac trunk and superior mesenteric artery in infants with necrotizing and superior mesenteric artery in infants with

4 Kempley ST, Gamsu HR, Vyas S, Nicolaides K. Effects of intrauterine growth retardation on postnatal visceral and cerebral blood flow velocity. Arch Dis Child 1991;66:111518.

5 Jawaheer G, Neal TJ, Shaw NJ. Blood culture volume and the detection of coagulase negative staphylococcal septicaemia in neonates. Arch Dis Child 1997;77:F157-8.

6 Schelonka RL, Chai MK, Yoder BA, Hensley D, Brockett RM, Ascher DP. Volume of blood required to detect common neonatal pathogens. F Pediatr 1996;129:275-8.

7 Ruokonen E, Takala J, Kari A, Saxen H, Mertsola J, Hansen E. Regional blood flow and oxygen transport in septic shock. Crit Care Med 1993;21:1296-303.

8 Coombs RC, Morgan MEI, Durbin GM, Booth IW, McNeish AS. Abnormal gut blood flow velocities in neonates at risk of necrotising enterocolitis. F Pediatr Gasneonates at risk of necrotising
troenterol Nutr 1992;15:13-19.

9 Siegel JH, Greenspan M, Del Guercio LRM. Abnormal vascular tone, defective oxygen transport and myocardial failure in human septic shock. Ann Surg 1967;165:504-17.

10 Duff JH, Groves AC, McLean A, LaPointe R, MacLean LD. Defective oxygen consumption in septic shock. Surgical Gynecology and Obstetrics 1969;128:1051-60.

11 Schumacker PT, Kazaglis J, Connolly HV, Samsel RW, O'Connor M-F, Umans JG. Systemic and gut oxygen extraction during endotoxaemia. Am f Respir Crit Care Med 1995;151:107-15. 